\title{
DETALLES SEDUCTORES RELEVANTES E IRRELEVANTES EN UN TEXTO EXPOSITIVO: EFECTOS SOBRE LACOMPRENSIÓN Y EL RECUERDO EN LECTORES CON BAJO CONOCIMIENTO PREVIO
}

\section{RELEVANT AND IRRELEVANT SEDUCTIVE DETAILS IN EXPOSITORY TEXT: EFFECTS ON RECALL AND COMPREHENSION IN READERS WITH LOW PRIOR KNOWLEDGE}

\author{
Gastón Saux \\ Universidad Católica Argentina \\ Natalia Irrazabal \\ CONICET, Argentina \\ Debora I. Burin \\ Universidad de Buenos Aires, Argentina
}

\begin{abstract}
Resumen: Se estudió el impacto de incluir detalles seductores sobre el recuerdo y la comprensión de un texto expositivo de ciencias, de contenidos poco familiares para los participantes. 94 estudiantes leyeron un texto expositivo sin detalles seductores, con un detalle seductor irrelevante (sin relación causal explícita con el resto del texto), o con un detalle seductor relevante (relacionado causalmente de modo explícito con el texto). La relevancia causal y el nivel de interés asociados al detalle fueron analizados en un pre-testeo inicial de los materiales. Los resultados indicaron que la condición que recibió el detalle en versión irrelevante tuvo más dificultades que el resto para recordar los contenidos y para contestar afirmaciones derivadas del texto. La condición que recibió el detalle seductor en versión relevante, por su parte, presentó el puntaje de recuerdo más alto. Estos resultados son interpretados atendiendo a su aplicación en el uso de textos en ámbitos educacionales.
\end{abstract}

Palabras Clave: comprensión de texto expositivo, detalle seductor, relevancia causal

\begin{abstract}
The effect of including seductive details on the recall and comprehension of an expository structured, scientific text was examined. 94 students with low prior knowledge on the subject read a text in one of following three conditions: without seductive details, with an irrelevant seductive detail (no causal relation with the rest of the message), or with a relevant seductive detail (with an explicit causal relation with the rest of the message). Causal relevance and the level of interest associated to the detail were analyzed prior to experimental activity. Results indicated that the Irrelevant Seductive Detail condition showed the poorest recall and the highest error rate in a sentence verification task. Relevant Seductive Detail condition, on the other hand, showed the best recall for text contents. These results are interpreted considering their potential applications in educational fields.
\end{abstract}

Keywords: expository text comprehension, seductive detail, causal relevance

\section{Introducción}

Comprender los textos producidos por otros es ciertamente tarea frecuente del científico y también de quien aprende ciencia. El presente trabajo se centra en las características de dichos textos y en su relación con los procesos cognitivos de extracción de significado a nivel discursivo. Se atiende especialmente al caso de los ámbitos educacionales, en los que lectores, todavía novatos en un cierto dominio temático, deben ser capaces de asimilar los mensajes transmitidos y construir representaciones coherentes de los mismos. En particular, el presente estudio se interesa por examinar cómo los agregados que tienen por objetivo interesar al lector pueden influir sobre la comprensión y el recuerdo del resto de la información.

Representada predominantemente en libros de texto, artículos científicos y de divulgación, la exposición es la forma textual que suelen tomar las explicaciones científicas. Un exposición se caracteriza por describir y explicar contenidos generalmente nuevos que se fundamentan en evidencia, haciendo referencia a fenómenos y sus partes componentes, sus funciones y sus mecanismos causales (León y Peñalba, 2002). De acuerdo con C. Padilla, S. Douglas y E. López (2007), la exposición se diferencia de otros tipos de texto tanto por sus funciones comunicativas como por sus rasgos formales predominantes. En el nivel de función

Correspondencia: Gastón Saux. Universidad Católica Argentina, Buenos Aires. Correo Electrónico: gsaux@psi.uba.ar 
comunicativa, el texto expositivo intenta hacer comprensible un tema considerado como válido o relativamente consensuado a nivel teórico, proponiendo una organización conceptual de modo "neutro", desde la posición de quien sabe a quien no sabe. De esta forma, la exposición se diferencia en la intención comunicativa respecto de textos que se centran en la mera presentación de información (descripciones), textos que intentan convencer al lector de una posición adoptada frente a un tema opinable (argumentaciones), textos que indican procedimientos (instrucciones) o textos que relatan episodios (narraciones). En el nivel formal, por su parte, la exposición se caracteriza por recurrir a enunciados genéricos, evidencias y relaciones lógicas entre las ideas, haciendo uso de marcas de impersonalidad que producen un efecto de objetividad, la preferencia del modo verbal indicativo y el uso de citas de autoridad.

A modo de ejemplo, considérese el siguiente extracto expositivo utilizado en un reciente estudio por J. Braasch, S. Goldman y J. Wiley (2013):

(...) Un gradiente de presión hace que las moléculas de aire se muevan de zonas de presión alta a zonas de presión baja. Este movimiento es experimentado como viento. Cuanto mayor sea el gradiente, más fuertes son los vientos que se producen (p. 577 , trad. propia).

Nótese la intención del texto por esclarecer o explicar el funcionamiento de un mecanismo a partir de una postura experta (nivel de función de uso). Para hacerlo, recurre a una relación lógica causal entre las ideas: el movimiento de moléculas de aires se produce porque éstas pasan de zonas de alta presión a zonas de baja presión (nivel formal-global), mediante el uso del presente indicativo y un estilo genérico, refiriendo a términos técnicos (nivel formallocal). La Tabla 1 presenta en modo resumido las características del texto expositivo.

De acuerdo con las investigaciones en el área, la exposición científica resulta menos familiar en contenido y en forma que otros tipos textuales, como la narración, de uso más frecuente en ámbitos cotidianos e informales (Wiley, Ash, Sanchez y Jaeger, 2011). A ello se suma que, dado que las exposiciones intentan alcanzar una explicación formal ideal, el tipo de relaciones que utilizan para organizar su prosa es de tipo abstracto (Meyer, 1985). Como resultado, la exposición científica corre el riesgo de
Tabla 1. Caracterización del texto expositivo, según Padilla et al. (2007)

\begin{tabular}{cl}
\hline Nivel de análisis & \multicolumn{1}{c}{ Caracterización } \\
\hline Función de uso & $\begin{array}{l}\text { Hacer comprensible un tema, explicar de } \\
\text { modo "neutro" }\end{array}$ \\
Formal - global & $\begin{array}{l}\text { Presentación de teorías, conceptos y } \\
\text { hechos a través de relaciones lógicas } \\
\text { abstractas }\end{array}$ \\
& $\begin{array}{l}\text { Uso de marcas de impersonalidad y citas de } \\
\text { autoridad, preferencia por el modo verbal } \\
\text { indicativo, uso de señalizaciones lógicas } \\
\text { entre las ideas }\end{array}$ \\
\hline
\end{tabular}

ser vista por quien intentará comprenderla como un material potencialmente "árido".

Frente a este escenario, resulta natural recurrir a estrategias que aumenten el atractivo de estos textos, en particular cuando se dirigen a lectores que no cuentan con un elevado nivel de experticia en el tema. Por ejemplo, F.J. Perales y J. Jiménez (2002) realizaron un análisis de 727 ilustraciones en nueve libros de texto de física de editoriales consolidadas en el mercado de habla castellana. Los investigadores hallaron la utilización dominante de fotografías e imágenes figurativas (en lugar de representaciones pictóricas abstractas) y una tendencia al uso de las imágenes para el realce decorativo. Esto constituye un potencial problema, puesto que se ha demostrado que la capacidad de una cierta información para atraer y motivar al lector, no presenta ninguna relación directa con su capacidad para promover la asimilación y adquisición de nuevos conocimientos (e.g. Levie \& Lentz, 1982; Mayer, Griffith, Jurkowitz y Rothman, 2008).

Una estrategia frecuente para capturar la atención del lector es agregar detalles seductores (DS) en el texto. Un DS se define como un agregado breve, interesante pero irrelevante, es decir, un agregado que busca capturar el interés del lector, pero sólo está tangencialmente relacionado con el resto del texto (Harp y Mayer, 1997). Desde el sentido común, la estrategia de agregar DS resulta lógica, pues se asume que el interés logrado ayudará a que el lector se centre en el mensaje, aumentando su predisposición para comprender el texto restante. Sin embargo, la investigación en el área ha señalado que la presencia de los DS se asocia con un efecto negativo, consistente en una disminución en las mediciones de recuerdo, comprensión y 
aprendizaje de los contenidos relevantes del texto, en comparación con materiales sin DS.

El apelativo DS fue introducido por primera vez por R. Garner, M. Gillingham y C. White (1989) quienes compararon el recuerdo de un texto expositivo con y sin anécdotas interesantes, pero poco relacionadas con el contenido principal. Por ejemplo, si el texto versaba sobre la vida solitaria o en grupo de los escarabajos, el texto podía incluir una referencia al particular modo que tienen los escarabajos de "girar en el aire" y producir "chasquidos" con este movimiento (p. 47). De modo sintético, los autores hallaron que: (1) los participantes calificaron este tipo de oraciones como muy interesantes pero poco relevantes, y (2) los participantes que leyeron estas oraciones recordaron menos ideas principales que los participantes que no las leyeron. Garner y colaboradores (1989) calificaron a este tipo de agregados tangenciales como detalles seductores (debido al interés despertado en los participantes) y concluyeron que su inclusión en el texto resultaría en un efecto negativo, también llamado efecto seductor, sobre el recuerdo de información textual importante (debido a las diferencias observadas entre las condiciones que leyeron o no la oración en cuestión). Posteriormente, el efecto seductor ha sido extendido a mediciones de comprensión inferencial y de transferencia de los contenidos aprendidos a partir del texto, encontrándose en materiales que versan sobre diversos temas, tanto en exposiciones escritas como orales. De modo característico, los participantes "seducidos" muestran un desempeño bajo con materiales que incluyen DS y no obstante evalúan estos materiales como más interesantes que los materiales de control sin DS (e.g. Harp \& Mayer, 1997; Ivanov, 2010; Lehman, Schraw, McCrudden \& Hartley, 2007; Park \& Lim, 2007; Sanchez \& Wiley, 2006).

En forma resumida, los estudios han hallado que los DS pueden distorsionar o interrumpir los procesos de comprensión, dirigiendo las capacidades de procesamiento de quien recibe el mensaje hacia los elementos anecdóticos del texto (Harp y Mayer, 1998; Mayer et al., 2008).

C. Harp y R. Mayer $(1997,1998)$ explicaron el efecto perjudicial de los DS a partir de la distinción propuesta por W. Kintsch (1980) entre interés cognitivo e interés emocional. El interés emocional se asocia con el mayor valor afectivo que, de modo típico, despierta cierta información (e.g. un dato curioso, un evento amenazante, etc.). El interés cognitivo, por otra parte, es el resultado de que el lector comprenda el texto estructuralmente y, como resultado, se implique en el proceso de lectura. De acuerdo con esta explicación, los DS aumentan el interés emocional, llevando al lector a sentir que disfruta de la actividad, pero no se asocian con el interés cognitivo. Los lectores seducidos tenderán a concentrar las capacidades limitadas de procesamiento en los detalles, en desmedro de la información menos seductora. Esta hipótesis ha sido extendida a mediciones de comprensión inferencial y de transferencia de los contenidos aprendidos a partir del texto, en los que los participantes muestran un desempeño bajo con materiales que incluyen DS y no obstante evalúan estos materiales como más interesantes que los materiales de control, sin DS (e.g. Mayer et al., 2008; Lehman et al., 2007; Park \& Lim, 2007; Peshkam; Mensink, Putnam, \& Rapp, 2011; Skolknik-Weisberg, Keil, Goodstein, Rawson y Gray, 2008).

Recientemente, G. Rey (2012) realizó un meta-análisis sobre 39 mediciones experimentales y halló un efecto significativo de los DS, tanto en las medidas de retención, como de comprensión y aprendizaje del texto, por lo que podría asumirse que el efecto perjudicial de los DS es sólido. Sin embargo, el concepto de DS no está exento de críticas (e.g. Goetz y Sadoski, 1995; Park, Moreno, Seufert, y Brünken, 2011; Rey, 2011; Towler, 2009). Parte de estas críticas se basan en una falta de criterio unificado sobre qué debe considerarse información seductora en cada caso. En concreto, un agregado es considerado DS cuando se observa la co-ocurrencia de tres atributos básicos: brevedad, alto interés y baja relevancia. La brevedad refiere a la extensión relativa del detalle respecto del resto del texto. El interés refiere a la curiosidad que despierta la información, asociada al grado de activación general (arousal) experimentado por el lector (Harp y Mayer, 1997). La relevancia refiere al grado de vinculación causal del detalle con el resto del texto (Harp y Mayer, 1998).

Estudios recientes han intentado paliar estas críticas, realizando análisis pormenorizados de los atributos que caracterizan a los DS y analizando su peso específico dentro del efecto general observado. El foco ha sido puesto en la extensión de los detalles verbales (Goetz y Sadoski, 1995; Park et al., 2011), así como en la manipulación del nivel de interés (bajo vs. alto) asociado a cada detalle (e.g. Ivanov, 2010; 
Mayer et al, 2008; Mikk y Kukemelk, 2010). En términos generales, estos estudios han hallado que: a) los DS no deberían exceder el treinta por ciento de la cantidad total de información de las condiciones control, puesto que el argumento del efecto seductor pierde fuerza luego de este límite. En particular, se ha planteado que la desviación de los procesos de comprensión con DS demasiado extensos podría atribuirse a que los lectores consideran que esta información debe ser recordada debido al espacio que ocupa (Park et al, 2011). Asimismo, estudios previos han propuesto que: (b) sólo los detalles con alto interés para los lectores resultan consistentes con el efecto seductor (Mayer et al. 2008). El estudio del interés cuenta con una larga tradición en la psicología educacional. Normalmente, se ha asumido que a mayor interés, mejor desempeño del aprendiz. No obstante, como se ha mencionado, en el caso de un DS el alto interés puede llevar a un bajo desempeño. Esta aparente contradicción puede resolverse a partir de la distinción propuesta por W. Kintsch (1980) entre interés emocional e interés cognitivo. El interés emocional resulta de información que apela a generar un estado afectivo en el lector (i.e. humor, miedo, etc.). El interés cognitivo, por su parte, resulta de la formación de una actitud positiva hacia la lectura que es causada por una comprensión profunda del material. Se ha propuesto que el interés asociado a un DS es específicamente de tipo emocional, mientras que el interés asociado a los procesos educacionales es de tipo cognitivo (Harp \& Mayer, 1997).

El papel específico de la irrelevancia del DS respecto del resto del texto, por otra parte, ha sido menos investigado. De acuerdo con Harp y Mayer (1997), la irrelevancia de un DS radica en que, aunque relacionado con el tópico general del texto, el detalle no presenta vínculos causales con el resto de la exposición. El establecimiento de vínculos causales (la detección de que algo es consecuencia de otra cosa) resulta particularmente importante para dotar de coherencia al texto expositivo (e.g. Graesser, McNamara, y Louwerse, 2003; Sanders y Mulder, 2012). Al presentar contenidos abstractos y técnicos, la exposición parece servirse especialmente de las relaciones causales, puesto que no siempre cuenta con otros parámetros básicos, como las dimensiones espacial, temporal, emocional e interpersonal, presentes en otro tipo de textos (Zwaan y Radvansky, 1998; León y Peñalba, 2002).
A pesar de la importancia del factor causal en la explicación de la comprensión del texto científico, muy pocos estudios han explorado este componente en relación con los DS. Algunos autores han sugerido que la irrelevancia de los DS produciría rupturas en la organización causal del texto expositivo, dificultando la construcción de representaciones coherentes de la información científica (Lehman et al., 2007; Schraw, 1998). S. Lehman et al. (2007), por ejemplo, presentaron a sus participantes un texto sobre la formación de los relámpagos en condición control (sin DS) o en condición seductora (el mismo texto más once oraciones seductoras). Los autores recolectaron los tiempos de lectura de las oraciones y luego solicitaron la producción de un ensayo sobre los contenidos del texto. En primer término, Lehman et al. hallaron que los participantes de la condición seductora tardaron más tiempo en leer las oraciones siguientes a los DS, en comparación con la condición control. En segundo término, los autores hallaron que la condición seductora presentó una comprensión significativamente más pobre de las relaciones causales propuestas en el texto (basada en un análisis causal del ensayo). Estos resultados fueron interpretados como evidencia de que la irrelevancia del detalle respecto del resto del texto (la falta de relación causal entre el DS y otros segmentos) había generado quiebres en la comprensión. Por esta razón, los lectores habían tenido que reconducir sus recursos de procesamiento luego de cada ruptura en la cohesión textual provocada por los DS, lentificando la lectura y generando representaciones menos organizadas. Lehman et al. (2007) concluyeron entonces que, si el texto fuese editado para que los DS presentasen mayor relación causal con el resto del texto, el efecto seductor podría mitigarse. Sin embargo, esta posibilidad no fue explorada.

Al respecto, sólo un estudio ha examinado los efectos de manipular la relevancia causal del DS, controlando el nivel de interés y la extensión del detalle. En concreto, I. Ivanov (2010) realizó un estudio en el que registró indicadores separados para el grado de interés, relevancia y otros factores asociados al material textual utilizado. Este autor halló que sólo el indicador de interés correlacionaba con el mejor recuerdo de la información seductora (a mayor interés consignado por el participante, mejor el recuerdo). Sin embargo, el estudio de Ivanov (2010) presenta dos limitaciones importantes. En pri- 
mer lugar, dicho estudio utilizó sólo una medida de recuerdo como indicador del resultado de la tarea de lectura. Desde la psicolingüística del texto, se ha señalado una diferencia importante entre las mediciones de recuerdo y de comprensión, así como la necesidad de recolectar más de una medición del proceso, debido a su complejidad (e.g. Irrazabal y Molinari, 2005). Además, las revisiones han propuesto que el efecto seductor es más fuerte para medidas de comprensión y aprendizaje que para medidas de recuerdo (Rey, 2012; Peshkam et al. 2011). Es posible entonces que la falta de significancia en los resultados reportados por Ivanov (2010), en relación con la relevancia causal del DS, sea atribuible al tipo de medida utilizada. En segundo lugar, la relevancia fue operacionalizada en el mencionado estudio como la calificación dada por los participantes en una escala tipo Likkert a cada oración, en referencia a la relación percibida por el lector entre esa oración y el resto del texto. Los problemas de los reportes subjetivos como indicadores del grado de comprensión de textos han sido consistentemente documentados (Wiley et al., 2011; McNamara, de Vega y O'Reilly, 2007), indicando la necesidad de complementar este tipo de mediciones con otros indicadores de la relevancia causal del DS para el resto del texto.

En síntesis, las investigaciones más recientes se han interesado por analizar qué componentes de los DS se asocian con el efecto seductor. Si bien muchos estudios han dado cuenta de la importancia del interés generado por el DS, el papel de la irrelevancia del detalle respecto del resto del texto ha sido menos estudiado. Las razones para estudiar el papel de la irrelevancia de los DS son dos. En primer lugar, en la mayoría de los estudios no es claro si el efecto negativo sobre la comprensión y el aprendizaje puede ser atribuido a la irrelevancia del DS, es decir, a su falta de relación con el resto texto, o se asocia principalmente con el alto interés que éstos despiertan. En segundo lugar, si se encontrase que el efecto negativo de los DS se ve mitigado al aumentar su relación con el resto del texto, el potencial daño del DS a la comprensión del resto del texto podría paliarse, conservando su capacidad para interesar al lector.

Teniendo esto en cuenta, el presente estudio se propuso examinar la comprensión y el recuerdo de un texto expositivo-científico que podía incluir o no un DS, variando su nivel de relevancia (i.e. vinculación causal) con el resto del texto y manteniendo fijo su nivel de interés. El supuesto fue que, si el efecto seductor es atribuible en parte a que la irrelevancia del detalle conduce a rupturas en la construcción de la coherencia, esto debería reflejarse en mayores dificultades para comprender y recordar el texto cuando los participantes recibiesen el detalle seductor en versión irrelevante, en comparación con el mismo detalle asociado de modo explícito con la organización causal del resto del texto.

\section{Método}

Análisis preliminar del interés. Previo a la realización del estudio, se realizó un pre-testeo del interés despertado en la población por el material que sería utilizado en el experimento, que consistió en un texto expositivo sobre el proceso físico-químico requerido para producir un aerogel de sílice (el contenido y estructura de los materiales será explicado en detalle en el apartado Materiales).

El objetivo de este pre-testeo fue determinar el nivel de interés asociado a las ideas que serían presentadas en el texto. Los participantes del pre-testeo fueron 35 estudiantes de $1 \mathrm{er}$. año de psicología. Siguiendo un procedimiento similar al utilizado por Mayer et al. (2008), se confeccionó un cuadernillo con las oraciones del texto, incluyendo también la que sería usada como DS, en orden aleatorio. Puesto que en esta instancia las oraciones fueron presentadas como ideas aisladas (y no como texto), algunas fueron levemente modificadas para conservar un sentido que no requiriese la referencia a otra oración. Por ejemplo, las anáforas pronominales o nominales fueron reemplazadas por el sustantivo que ocupaba el lugar del antecedente. 18 participantes recibieron las oraciones resultantes en una determinada secuencia y los 17 restantes en la secuencia inversa. Se indicó a los participantes que leerían una serie de enunciados relativos a un tema científico y se les solicitó que, independientemente de su conocimiento del tema, calificasen cada oración en función de cuán interesante le resultaba la idea expresada, indicando en una escala un valor entre 1 (nada interesante) a 7 (muy interesante).

Se computaron los promedios de nivel de interés asignado para cada oración. En promedio, los participantes encontraron a las ideas del texto de nada a levemente interesantes $(M=2.6, S D=.46)$, mientras que la idea que sería utilizada como DS en el experimento fue 
calificada con un puntaje superior al doble del promedio del resto del material (de levemente a algo interesante, $M=5.5, S D=.98$ ).

Se asumió entonces que, en el experimento, el DS produciría mayor interés en los participantes que el resto del texto, por lo que, de encontrar diferencias asociadas a variaciones de la relevancia del detalle, éstas no podrían ser atribuidas directamente al nivel de interés, que, aunque alto, se mantuvo fijo en todas las condiciones. La Tabla 2 muestra, a modo de ejemplo, las oraciones calificadas como más y menos interesantes por los participantes.

Tabla 2. Puntaje promedio de interés, desvio estándar y ejemplo de las oraciones calificadas como más y menos interesantes por los participantes en el pre-testeo

\begin{tabular}{lll}
\hline Interés alto $(5.5 \pm .98)$ & Interés bajo $(1.5 \pm 1.01)$ \\
\hline \multirow{4}{*}{ Enunciado sus muchas } & \\
& $\begin{array}{l}\text { Enticaciones, la Nasa ha } \\
\text { utilizado los aerogeles } \\
\text { para atrapar polvo compuesto por una } \\
\text { cósmico en misiones } \\
\text { espaciales. }\end{array}$
\end{tabular}

Puntaje de interés expresado como media \pm desvío estándar.

\section{Participantes}

Participaron voluntariamente 94 estudiantes de Psicología de primer año de la carrera (Edad $\mathrm{M}=22.43$, DS = 6.21). La muestra estuvo compuesta por 64 mujeres $(68 \%)$ y 30 varones $(32 \%)$. Los participantes fueron informados acerca del propósito del estudio y del procedimiento experimental y prestaron su consentimiento por escrito. Una vez concluido el experimento, las hipótesis y los resultados esperados fueron transmitidos grupalmente.

\section{Diseño y Materiales}

Se crearon tres condiciones, en las que se varió la modalidad de presentación del texto:

1. Texto sin DS (condición control),

2. Texto con un DS sin relación causal con el resto del material (condición DS irrelevante), y

3. Texto con unDS causalmente relacionado con el resto del material (condición DS relevante).

Texto. El material consistió en un texto de estructura expositiva sobre el proceso físico químico requerido para producir un aerogel de sílice. El texto fue originalmente extraído de una revista de divulgación científica y fue posteriormente adaptado en longitud y estructura de la exposición con el objeto de ser utilizado en el presente estudio. Adicionalmente, se reordenaron algunas oraciones, se agregó información de otras fuentes y se quitó el título. La versión final del texto tuvo una extensión de 217 palabras (sin contar el DS), no incluyó título ni sub-títulos y fue dividido en 22 líneas, presentadas en dos páginas. Cada página presentó sub-temas específicos dentro del tópico principal. La primera página (12 líneas) tuvo como objetivo introducir el tema a partir de una breve definición y descripción de los aerogeles (4 líneas) y explicar los pasos del proceso de secado utilizado para la creación de los mismos (8 líneas). En la segunda página (10 líneas) se expuso el concepto de punto crítico (niveles específicos de temperatura y presión requeridos para crear aerogeles). La Tabla 3 presenta el material utilizado.

En las condiciones seductoras, se agregó un DS, consistente en dos oraciones relativas al uso de los aerogeles en misiones espaciales por la NASA (ver tabla 1). La información fue considerada DS debido a: (1) su corta longitud (menos del $30 \%$ del material original), (2) el elevado interés consignado por los participantes por esta información (ver Pre-testeo de los materiales más arriba), y (3) su poca relación con el resto del texto, centrado en la explicación del proceso físico químico de obtención de los aerogeles. Medido en cantidad de palabras, el detalle agregado representó entre el $14 \%$ (DS Relevante) y 16\% (DS Irrelevante) de la extensión total del material (32 y 34 palabras, respectivamente). EI DS fue presentado siempre en la quinta línea de la primera página y representó el $6 \%$ de la extensión total del material (medido en cantidad de palabras). La decisión de incluir el DS en la parte inicial del texto se basó en estudios previos que sugieren que el efecto seductor es más claro cuando los detalles se encuentran al comienzo del texto (Harp y Mayer, 1998; Rowland-Bryant et al., 2008). No se incluyeron DS en la segunda página, con el objeto de poder comparar el posterior recuerdo y comprensión del texto como un todo (ambas páginas juntas), o en segmentos con y sin información seductora (pág. 1 vs. pág. 2).

La relevancia del DS fue manipulada creando dos versiones similares de la información, 
Tabla 3. Texto empleado en el estudio

\begin{tabular}{|c|c|}
\hline $\begin{array}{l}\text { Página 1, segmento } 1 \\
\text { (líneas } 1 \text { a } 4 \text { ) }\end{array}$ & $\begin{array}{l}\text { Los aerogeles son, hoy por hoy, uno de los materiales menos densos y más livianos que se } \\
\text { conocen. } \\
\text { Deben su liviandad a una gran porosidad. } \\
\text { Alrededor del } 95 \% \text { del volumen total de un aerogel está compuesto por espacios llenos de aire. }\end{array}$ \\
\hline DS irrelevante & $\begin{array}{l}\text { Entre sus muchas aplicaciones, la Nasa ha utilizado los aerogeles para atrapar polvo cósmico en } \\
\text { misiones espaciales. } \\
\text { En un futuro muy cercano, seguramente habrá muchas más aplicaciones y avances en la tecnolo- } \\
\text { gía de los aerogeles. }\end{array}$ \\
\hline DS relevante & $\begin{array}{l}\text { Debido a su estructura compuesta por una gran cantidad de pequeños poros, entre sus muchas } \\
\text { aplicaciones, la Nasa ha utilizado los aerogeles para recolectar pequeñas partículas de polvo } \\
\text { cósmico en misiones espaciales. }\end{array}$ \\
\hline $\begin{array}{l}\text { Página 1, segmento } 2 \text { (líneas } \\
5 \text { a 12) }\end{array}$ & $\begin{array}{l}\text { Los aerogeles son hechos a partir de un proceso conocido como "secado supercrítico". } \\
\text { El secado supercrítico comienza con un gel común, normalmente de sílice, } \\
\text { que está compuesto por una estructura de poros y líquido. } \\
\text { El gel común se licúa con gas, logrando extraer la porción líquida del gel. } \\
\text { El fluido resultante es secado con un proceso especial. } \\
\text { El proceso lleva la presión y temperatura del fluido a un nivel específico, llamado "nivel crítico". } \\
\text { La sustancia resultante es el aerogel. }\end{array}$ \\
\hline $\begin{array}{l}\text { Página 2, segmento } 3 \text { (líneas } \\
12 \text { a 22) }\end{array}$ & $\begin{array}{l}\text { El "nivel crítico" de una sustancia separa sus fases líquida y gasesosa. } \\
\text { Una sustancia que alcanza su nivel crítico presenta características cruzadas entre las de un } \\
\text { líquido y las de un gas. } \\
\text { El secado supercrítico permite alcanzar y superar este nivel. } \\
\text { Casi todo el líquido del gel original es reemplazado por aire, } \\
\text { sin que se pierda la estructura en el material resultante. } \\
\text { El aerogel final está constituido por poros microscópicos y aire. } \\
\text { Estos microporos están conectados entre sí, formando una red continua. } \\
\text { Puesto que esta red se sostiene a sí misma, } \\
\text { el aerogel se considera un sólido. }\end{array}$ \\
\hline
\end{tabular}

pero modificando su relación causal explícita con el resto del texto. En la condición DS irrelevante, las dos oraciones (el DS) fueron presentadas de forma descontextualizada, sin hacer referencia explícita a su relación con el resto del texto. En la condición DS relevante, se varió una de las oraciones para, de modo explícito, vincular causalmente la descripción de la estructura porosa del areogel con su utilidad para capturar polvo espacial. La variación consistió en reemplazar una de las dos oraciones del DS por otra que permitiese ver la relación entre la estructura físico-química del aerogel y sus posibilidades de uso como recolector de polvo (ver Tabla 2).

Análisis de la relación causal DS - resto del texto. Previo a la realización del estudio, las dos versiones seductoras del texto fueron analizadas y comparadas con el software Expository Text Analysis Tool (ETAT, Vidal-Abarca et al., 2002). EI ETAT es un software que permite analizar el grado de coherencia de los textos expositivos. Su uso para la revisión de textos expositivos en español ha sido documentado en diferentes oportunidades (e.g. Gilabert, Martinez y Vidal-Abarca, 2005). Al igual que programas y modelos similares de análisis de textos (e.g. Graesser, 1981; van den Broek, Young, Tzeng y Linderholm, 1998) el ETAT busca representar la organización semántica o conceptual de un texto. El procedimiento de análisis en el ETAT sigue tres pasos: la segmentación del texto en nodos (oraciones simples o compuestas subordinadas), la clasificación de los nodos en una de tres categorías ("estado", "evento" o "meta"; en el caso de oraciones subordinadas, la cláusula principal es la que guía la clasificación) y el establecimiento de relaciones entre los distintos nodos del texto por medio de la comparación de pares de nodos sobre la base de seis categorías o tipos de relaciones ("inicia", "resultado", "razón", "causa", "descripción" y "ejemplo") y sobre la base del carácter explícito (indicado por el texto) o implícito (no indicado) de la relación (para una descripción pormenorizada de la herramienta y su aplicación, se sugiere ver Vidal-Abarca et al., 2002).

Una vez realizado el análisis, el ETAT produce automáticamente varios estadísticos descriptivos de los nodos del texto y sus interrelaciones, junto con un grafo (representación gráfica) de los mismos. En el presente estudio, el material textual fue analizado por separado, atendiendo a las dos versiones seductoras. En términos generales, los resultados arrojados por el ETAT mostraron una buena relación entre los nodos del texto, con una media de entre 
2.4 (condición DS irrelevante) y 3 (condición DS relevante) relaciones por nodo. En relación con el DS, los análisis confirmaron diferencias importantes en el grado de conexión causal del DS respecto del resto del texto entre las condiciones relevante e irrelevante. En concreto, en la condición DS irrelevante, los dos nodos del DS presentaron el número más bajo de relaciones con el resto del texto (1 relación por cada nodo, en promedio). En la condición DS relevante, por su parte, la cantidad de relaciones para los nodos del DS subió a 5.5 relaciones, en promedio. De esta manera, se buscó garantizar la mayor o menor relevancia asumida para el DS en cada condición. La Tabla 4 muestra los estadísticos producidos por el ETAT para el texto en sus dos versiones seductoras.

Tabla 4. Estadísticos producidos por el ETAT para el grado de relación del texto y los DS, en versión DS relevante e irrelevante

\begin{tabular}{|c|c|c|}
\hline & $\begin{array}{l}\text { Condición DS } \\
\text { irrelevante }\end{array}$ & $\begin{array}{l}\text { Condición DS } \\
\text { relevante }\end{array}$ \\
\hline Cantidad de nodos & 20 & 20 \\
\hline $\begin{array}{l}\text { Cantidad de relaciones } \\
\text { entre nodos }\end{array}$ & 24 & 30 \\
\hline Conexiones por nodo & $2.4 \pm 0.9$ & $3 \pm 1.2$ \\
\hline $\begin{array}{l}\text { Cantidad de relaciones } \\
\text { causales (\%) }\end{array}$ & $9(37.5 \%)$ & $10(33.3 \%)$ \\
\hline Conexiones por nodo del DS & $1 \pm 0$ & $5.5 \pm 2.1$ \\
\hline $\begin{array}{l}\text { Cantidad de relaciones } \\
\text { causales entre los nodos } \\
\text { del DS y el resto del texto (5) }\end{array}$ & $0(0 \%)$ & $3(10 \%)$ \\
\hline
\end{tabular}

Medidas de recuerdo y comprensión. Se recolectaron dos indicadores post-lectura del recuerdo y la comprensión y un indicador del nivel de conocimiento previo. Para obtener estos indicadores se preparó un cuadernillo con las siguientes consignas:

1. Redactar lo relativo al recuerdo

2. Redactar lo relativo a escala de valores

3. Redactar lo relativo a la tarea de verificación.

En primer lugar, el cuadernillo solicitó a los participantes escribir toda la información que recordasen del texto (10 líneas máx.). En segundo lugar, se solicitó a los participantes que seleccionasen en una escala con valores entre 1 ('nada') y 9 ('todo'), el nivel de conocimiento previo sobre los contenidos del texto. Finalmente, el cuadernillo presentó una tarea de verificación de 16 frases. Los participantes debían responder si la frase presentada era verdadera, falsa, o si no sabían la respuesta. La mitad de las afirmaciones se basó en contenido de la primera página del texto, la otra mitad se basó en contenidos de la segunda página. Adicionalmente, la mitad fueron afirmaciones literales (idénticas a oraciones del texto), y la otra mitad fueron afirmaciones inferidas (información que puede derivarse del texto aunque no esté mencionada explícitamente). De este modo, los participantes debieron contestar cuatro afirmaciones literales y cuatro afirmaciones inferidas para cada página del texto. El orden de los ítems presentados en la tarea de verificación fue variado en las presentaciones. Se crearon 8 sets, compuestos de 2 ítems cada uno, seleccionados al azar. Se rotó el orden de los sets, creando 8 combinaciones de orden. Cada participante recibió los ítems en alguna de las combinaciones.

\section{Procedimiento}

Se realizaron sesiones de grupos de 30 participantes aproximadamente. Primero, los participantes leyeron el texto en alguna de las tres condiciones. Luego, los participantes entregaron el texto y recibieron a cambio el cuadernillo junto con las instrucciones para las actividades.

\section{Resultados}

Los datos fueron analizados con el Paquete Estadístico para Ciencias Sociales -SPSS- versión 17 para Windows (IBM, 2009). El punto de corte para la significancia de los $p$ valores reportados fue de .05 .

Familiaridad con el tema: En primer lugar, se analizó el grado de familiaridad con el tema reportado por los participantes en la escala de 1 a 9 , incluida en el cuadernillo. El texto recibió, en líneas generales, puntajes bajos en familiaridad, tanto en la condición Sin DS $(M=1.18, D S=.58)$, como en las condiciones DS Irrelevante $(M=1.7, D S=1.4)$ y DS Relevante $(M=1.64, D S=1.3)$. A continuación, se analizó el efecto de la Modalidad de Presentación del material sobre los puntajes de Familiaridad, mediante un análisis de varianza de un factor para grupos independientes. El efecto del Tipo de Presentación no fue significativo $[F$ $\left.(2,91)=1.99, M S_{e}=1.4, p=.14\right]$. Por lo tanto 
se asumió que los grupos: a) no eran expertos en el tema presentado por el texto, y b) no diferían entre sí en el nivel de conocimiento previo reportado.

Tarea de recuerdo: Para poder analizar los protocolos de recuerdo, se dividió el texto en tres segmentos y se identificaron las ideas contenidas en cada segmento. Para el análisis del recuerdo, se asignó un punto por cada idea presente en el protocolo y 0 por cada idea no mencionada. En el primer segmento (introducción del texto, cuatro primeras líneas de la primera página), se identificó una idea. En los dos segmentos restantes (8 líneas restantes de la primera página y 10 líneas de la segunda página) se identificaron 5 ideas, respectivamente. Adicionalmente, se registró el recuerdo del DS.

Se realizaron ANOVAs de una vía sobre la cantidad de ideas recordadas, tomando la Modalidad de Presentación del Material como factor. Los análisis mostraron un efecto altamente significativo para el recuerdo del DS $[F(2,91)=57.2$, $M S e=.11, p=.0001]$. Comparaciones post-hoc pareadas (HSD de Tukey) revelaron que, como era esperable, las condiciones DS irrelevante y DS relevante mencionaron significativamente más veces el DS en sus protocolos, en comparación con la condición sin DS $(p<.0001)$.
No se encontraron diferencias entre ambas condiciones seductoras $(p=.86)$. Respecto al recuerdo del resto del texto, los análisis indicaron un efecto significativo sólo para el segundo segmento de la primera página del texto (la parte del texto en la que se incluyó el DS) $[F(2,91)=3.2, M S e=1.5, p=.04]$. La observación de las medias indicó que la condición DS Relevante presentó el promedio de recuerdo más alto $(M=2.1, S D=1.4)$, seguida por la condición Sin DS $(M=1.9$, $S D=1.38$ ), seguida por la condición $D S$ Irrelevante $(M=1.3, S D=.86)$. Sin embargo, los análisis post-hoc indicaron que sólo la comparación entre extremos (DS Irrelevante vs. DS Relevante) resultó significativa. En otros términos, el grupo que recibió el DS sin relación explícita con el resto del texto recordó menos unidades de la página que incluyó dicho detalle, en comparación con el grupo que recibió el mismo detalle pero integrado causalmente al resto del texto $(p=.04)$. Las comparaciones restantes para el segmento no mostraron diferencias significativas. La Tabla 5 presenta los estadísticos descriptivos del recuerdo y del DS por segmento para las tres condiciones experimentales.

Verificación de frases. Se realizaron ANOVAs de una vía con la Modalidad de Pre-

Tabla 5. Medias y desvios estándar para los puntajes de recuerdo del DS y del resto del texto por segmento y página, según Modalidad de Presentación del texto

\begin{tabular}{|c|c|c|c|}
\hline \multirow[b]{2}{*}{ Segmento y página del texto } & \multirow[b]{2}{*}{ Condición } & \multicolumn{2}{|c|}{ Recuerdo } \\
\hline & & $M$ & $S D$ \\
\hline \multirow{3}{*}{$\begin{array}{l}\text { Segmento } 1 \text {, página } 1 \\
\text { [puntuación máx. 1] }\end{array}$} & $\operatorname{Sin} \mathrm{DS}$ & .63 & .49 \\
\hline & DS Irrelevante & .72 & .45 \\
\hline & DS Relevante & .77 & .61 \\
\hline \multirow{3}{*}{$\begin{array}{l}\text { Segmento 2, página } 1 \\
\text { [puntuación máx. 5] }\end{array}$} & Sin DS & 1.93 & .98 \\
\hline & DS Irrelevante & $1.39^{a}$ & .86 \\
\hline & DS Relevante & $2.16^{a}$ & 1.4 \\
\hline \multirow{3}{*}{$\begin{array}{l}\text { Segmento } 3 \text {, página } 2 \\
\text { [puntuación máx. 5] }\end{array}$} & Sin DS & 1.36 & 1.08 \\
\hline & DS Irrelevante & 1.22 & 1.23 \\
\hline & DS Relevante & .8 & 1.09 \\
\hline \multirow{3}{*}{$\begin{array}{l}\text { Total (ambas páginas) } \\
\text { [puntuación máx. 11] }\end{array}$} & $\operatorname{Sin} D S$ & 3.36 & 2.38 \\
\hline & DS Irrelevante & 3.48 & 1.6 \\
\hline & DS Relevante & 4.16 & 2.6 \\
\hline \multirow{3}{*}{ Recuerdo del DS } & Sin DS & $.0^{\mathrm{a}}$ & .0 \\
\hline & DS Irrelevante & $.81^{\mathrm{a}}$ & .39 \\
\hline & DS Relevante & $.77^{\mathrm{a}}$ & .42 \\
\hline
\end{tabular}


sentación del material como factor sobre la cantidad de errores en la tarea de verificación de frases, teniendo en cuenta el carácter literal o inferido de la afirmación y si la misma había sido formulada sobre la base de los contenidos de la primera o la segunda página del texto. Todos los análisis fueron realizados sobre las proporciones de errores. Se encontró un efecto significativo de la Modalidad del Texto sobre la cantidad de errores en la verificación de frases asociadas a la primera página del texto $\left[F(2,91)=3.2, M S_{e}=.04, p=.04\right]$. Las pruebas post-hoc mostraron que la condición Sin DS presentó una cantidad significativamente menor de errores en la verificación de enunciados $(M=.14, S D=.16)$, en comparación con la condición DS Irrelevante $(M=.26$, $S D=.2, p=.05$ ). La condición DS Relevante presentó una tasa de errores intermedia $(M=.16, S D=.17)$, que no alcanzó a diferenciarse de las dos condiciones restantes $(p>.05)$. Los análisis sobre el total de los errores, y sobre los errores para la segunda página no mostraron efectos significativos $(p>.05)$.

\section{Discusión}

El objetivo del presente estudio fue examinar la comprensión y el recuerdo de un texto expositivo de contenidos científicos que podía incluir o no un DS, variando su nivel de relevancia (i.e. vinculación causal) con el resto del texto y manteniendo fijo el nivel de interés del detalle. El supuesto fue que, si la irrelevancia juega algún papel en el efecto negativo asociado a los DS, esto debería reflejarse en mayores dificultades para comprender y recordar el texto en condiciones en que el texto presentara el DS de modo irrelevante, es decir, con poca relación con el resto del texto, en comparación con el mismo detalle asociado causalmente de modo explícito con la organización del texto.

En términos generales, los resultados obtenidos permiten sostener el efecto negativo sobre el procesamiento del texto, atribuible a detalles interesantes e irrelevantes (DS). Sin embargo, la manipulación de la contextualización causal del DS parece haber reducido sustancialmente dicho efecto negativo. Esta afirmación se basa en que, mientras que la condición que leyó el texto con el DS en versión irrelevante presentó el índice más bajo de recuerdo y la mayor proporción de errores en la tarea de verificación, los participantes en la condición DS Relevante tuvieron el mejor desempeño en el recuerdo de los contenidos del texto.

En relación con la tarea de recuerdo, cabe destacar que, aunque ambas condiciones seductoras tuvieron una tasa elevada y similar de recuerdo del DS, presentaron diferencias en el recuerdo del resto del material. En concreto, el mejor recuerdo de la condición DS Relevante se focalizó exclusivamente en la sección del material en la que se incluyó el DS (segundo segmento de la primera página) y no en los demás segmentos texto. Este patrón de resultados sería consistente con la hipótesis de que la irrelevancia de los DS produce rupturas en la cohesión del texto, y esto afecta los procesos de organización mental de la información y, en consecuencia, su recuerdo (Lehman et al., 2007). En concreto, los presentes resultados permitirían sostener que el efecto negativo sobre el recuerdo, atribuible a rupturas en la cohesión textual, queda restringido a los sectores del material en los que se incluye el DS, sin observarse efectos sobre otros pasajes textuales.

Cabe destacar, a su vez, la falta de diferencias hallada entre la condición control sin DS y las condiciones seductoras en la tarea de recuerdo. Se considera que este dato requiere de mayor análisis, puesto que la observación de las medias indicó una tendencia de la condición control a ubicarse en una posición a mitad de camino entre las condiciones seductoras. Por lo tanto, si manteniendo dicha tendencia, se lograsen establecer diferencias significativas entre el desempeño de la condición control y una o ambas condiciones seductoras, podría afirmarse que la mayor relevancia del DS no sólo reduce el efecto negativo, sino que supone además una facilitación del recuerdo, en comparación con una condición sin DS. Futuras investigaciones deberán establecer el peso de estas consideraciones.

Con respecto a la tarea de verificación, los resultados indican que la condición DS Irrelevante cometió más errores al contestar afirmaciones de la primera página (la página que incluyó el DS), en comparación con la condición sin DS. Estos datos resultan consistentes con el desempeño de la condición DS Irrelevante, observado en la tarea de recuerdo. Al igual que en aquella medida, se encontró que la condición que leyó el DS sin nexos causales explícitos con el resto del texto fue la que tuvo mayores dificultades para realizar la tarea. Sin embargo, cabe preguntarse por qué el patrón 
de mejor desempeño en esta tarea (atribuido a la condición Sin DS) no se corresponde con el patrón de mejor desempeño observado en la tarea de recuerdo (atribuido a la condición DS Relevante). Una posible explicación sería que la tarea de recuerdo podría estar reflejando mejor los efectos del interés despertado por el DS. En concreto, el testeo previo de los materiales indicó que los participantes encontraron el agregado seductor mucho más interesante (más del doble) que el resto de la información. Entonces, sería posible que, en la tarea de recuerdo, los participantes en las condiciones seductoras hayan tenido facilitado el acceso a la información sobre el DS. Esto sería compatible, por otra parte, con el alto nivel de recuerdo del detalle en ambas condiciones seductoras. En el caso de la condición DS Relevante, las conexiones explícitas entre esta información y el resto, podría haber llevado a los participantes, además, a recordar la información asociada al detalle. De asumirse esta explicación, debería aceptarse, sin embargo, que el efecto del elevado interés del detalle no fue suficiente para producir una mejora sobre la verificación de afirmaciones, en comparación con la condición control (sin DS).

Finalmente, debe tenerse en cuenta que los resultados y conclusiones teóricas derivadas de este estudio sólo deberían generalizarse, como lo indica el análisis del índice de conocimiento previo recolectado durante la actividad, a lectores con bajo conocimiento previo en el tema. Desde la psicolingüística del texto, se ha señalado que la alta coherencia de un texto (en el sentido en que explicita muchas de las relaciones entre sus ideas) no siempre es mejor. En el caso de lectores expertos, se ha observado que un grado elevado de coherencia textual puede llevar a un procesamiento más superficial (e.g. McNamara, Kintsch, Songer y Kintsch, 1996). Por lo tanto, sería posible que el aumento de la relevancia del DS produjese efectos muy diferentes a los aquí reportados, si los lectores fuesen expertos en el tema.

\section{Conclusiones}

Los resultados del presente trabajo indican que la presentación de DS modula el procesamiento de textos expositivos de contenidos científicos, en lectores con bajo conocimiento previo. En particular, la relevancia del DS constituiría un factor a tener en cuenta a la hora de diseñar textos en contextos educacionales que incluirán agregados breves e interesantes, con miras a capturar la atención de un aprendiz todavía novato en el tema.

A partir de los resultados obtenidos, puede afirmarse que el efecto negativo de tales detalles puede mitigarse variando la relevancia de los mismos en función del resto del mensaje. Esto se debería, de acuerdo a los resultados obtenidos, a que parte de la influencia de los DS sobre la actividad mental radica en la falta de conexión explícita de dichos detalles respecto a la organización del texto. La explicitación de conexiones causales entre el agregado interesante y el resto del mensaje permitiría, entonces, reducir su potencial influencia.

En los contextos aplicados, la eliminación de agregados breves pero interesantes de las exposiciones científicas conlleva el riesgo de desinteresar al aprendiz, que tiende a ver al libro de texto científico como poco interesante y aburrido (e.g. Hidi y Renninger, 2006). Teniendo esto en cuenta, el presente trabajo de investigación ha intentado ser un aporte al estudio de los factores que modulan el impacto de incluir DS en el texto científico.

\section{Referencias}

Braash, J., Goldman, S. \& Wiley, J (2003). Effects of working memory capacity on learning from ilustrated texty. Memory \& Cognition, 34(2), 344-355.

Garner, R., Gillingham, M. G., \& White, C. S. (1989). Effects of "seductive details" on macroprocessing and microprocessing in adults and children. Cognition and Instruction, 6, 41-57.

Gilabert, R., Martinez, G., \& Vidal-Abarca, E. (2005). Some good texts are always better: Text revision to foster inferences of readers with high and low prior background knowledge. Learning and Instruction 15, 45-68.

Goetz, E., \& Sadoski, M. (1995). The perils of seduction: Distracting details or incomprehensible abstractions? Reading Research Quarterly, 30, 518-519.

Graesser, A. (1981). Prose comprehension beyond the word. New York: Springer-Verlag.

Graesser, A., McNamara, D. \& Louwerse, M. (2003). What do readers need to learn in order to process coherent relations in narrative and expository text. En A. Sweet \& C. Snow (Eds.) Rethinking reading comprehension (pp 82-98). New York: Guilford Publications.

Harp, S. F., \& Mayer, R. E. (1997). The role of interest in learning from scientific text and illustrations: On the distinction between emotional and cognitive interest. Journal of Educational Psychology, 89, 92-102.

Harp, S. F., \& Mayer, R.E. (1998). How seductive details do their damage: A theory of cognitive interest in science learning. Journal of Educational Psychology, 90, 414-434.

Hidi, S., \& Renninger, K. A. (2006). The four-phase model of interest development. Educational Psychologist, 41(2), 111-127. 
IBM (2009) Statistical Package for Social Science (18) [Software de computación]. Chicago: SPSS Inc.

Irrazabal, N. \& Molinari, C. (2005). Técnicas experimentales en la investigación de la comprensión del lenguaje. Revista Lat. de Psicología, 37, 581-594.

Ivanov, I. (2010). Predictors of recall and reading time for seductive and nonseductive text segments. (Tesis de maestría no publicada), University of Nevada, USA.Kintsch, W. (1980). Learning from text, levels of comprehension, or: Why anyone would read a story anyway. Poetics, 9, 87-98.

Lehman, S., Schraw, G., McCrudden, M. T., \& Hartley, K. (2007). Processing and recall of seductive details in scientific text. Contemporary Educational Psychology, 32, 569-587.

León, J.A. \& Peñalba, G. (2002). Understanding causality and temporal sequence in scientific discourse. En J. Otero, J. A. León \& A. Graesser (Comps.), The psychology of science text comprehension (pp 155-178). Mahwah, New Jersey: Lawrence Erlbaum Associates.

Levie, W. H. y Lentz, R. (1982). Effects of text illustrations: A review of research. Journal of Educational Communication and Technology, 30, 195-232.

Mayer, R., Griffith, E., Jurkowitz, I., \& Rothman, D. (2008) Increased interestingness of extraneous details in a multimedia science presentation leads to decreased learning. Journal of Experimental Psychology: Applied, 14, 329-339.

McNamara, D., de Vega, M. \& O'Reilly, T. (2007). Comprehension skill, inference making, and the role of knowledge. En F. Schmalhofer \& C.A. Perfetti (Eds.), Higher level language processes in the brain: Inference and comprehension processes (pp. 233253). Mahwah, NJ: Erlbaum.

McNamara, D., Kintsch, E., Songer, N.B., \& Kintsch, W. (1996). Are good texts always better? Cognition and Instruction, 14, 1-43.

Meyer, B. (1985). Prose analysis: Purposes, procedures, and problems. En B.K. Britton \& J. Black (Eds.), Analyzing and understanding expository text, (pp. 269-304). Hillsdale, New Jersey: Lawrence Erlbaum Associates.

Mikk, H., \& Kukemelk, H. (2010). The relationship of text features to the level of interest in science texts. Trames, 14, 54-70.

Padilla, C., Douglas, S. \& López, E. (2007). Yo expongo. Taller de prácticas de comprensión y producción de textos expositivos. Córdoba: Comunicarte.

Park, S., \& Lim, J. (2007). Promoting positive emotion in multimedia learning using visual illustrations. Journal of Educational Multimedia and Hypermedia, 16(2), 141-162.

Park, B., Moreno, R., Seufert, T., \& Brünken, R. (2011). Does cognitive load moderate the seductive details effect? A multimedia study. Computers in Human Behavior, 27, 5-10.
Perales, F. J. \& Jiménez, J. (2002). Las ilustraciones en la enseñanza-aprendizaje de las ciencias. Análisis de libros de texto. Enseñanza de las Ciencias, 20(3), 369-386.Peshkam, A., Mensink, M. C., Putnam, A. L., \& Rapp, D. N. (2011). Warning readers to avoid irrelevant information: When being vague might be valuable. Contemporary Educational Psychology, 36, 219-231.

Peshkam, A., Mensink, M. C., Putnam, A. L., \& Rapp, D. N. (2011). Warning readers to avoid irrelevant information: When being vague might be valuable. Contemporary Educational Psychology, 36, 219-231.

Rey, G. D. (2011). Seductive details in multimedia messages. Journal of Educational Multimedia and Hypermedia, 20, 283-314.

Rey, G. D. (2012). A review of research and a meta-analysis of the seductive detail effect. Educational Research Review, 7, 216-237.

Rowland-Bryant, E., Skinner, C. H., Davis-Richards, K., Saudargas, R., \& Robinson, D. H. (2008). An investigation of placement and type of seductive details: The primacy effect of seductive details on text recall. Research in the Schools, 15, 80-90.

Sanders, T.J.M. \& Mulder, G. (2012). Causal relations and levels of discourse representation. Discourse Processes, 49(6), 501-522.

Sánchez, C.A., Wiley, J. (2006). The influences of text and reader characteristics of learning from refutations science texts. Journal of Educational Psychology, 105, 561-568.

Schraw, G. (1998). Processing and recall differences among seductive details. Journal of Educational Psychology, 90, 3-12.

Skolnick-Weisberg, D., Keil, F., Goodstein, J., Rawson, E., \& Gray, J. (2008). The seductive allure of neuroscience explanations. Journal of Cognitive Neuroscience, 20, $470-477$

Towler, A. (2009). Effects of trainer expressiveness, seductive details, and trainee goal orientation on training outcomes. Human Resource Development Quarterly, 20, 65-84.

van den Broek, P. , Young, M., Tzeng, Y. \& Linderholm, T. (1998). The landscape model of reading. En H. van Oostendorp \& S. Goldman (Eds.), The construction of mental representations during reading (pp. 71-98). Mahwah, New.Jersey: Lawrence Erlbaum.

Vidal-Abarca, E., Reyes, H., Gilabert, R., Calpe, J., Soria, E. \& Graesser, A. (2002). ETAT: Expository Text Analysis Tool. Behavior Research Methods, Instruments and Computers, 34, 93-107.

Wiley, J., Ash, I.K., Sanchez, C.A. \& Jaeger, A.J. (2011). Clarifying readers' goals for learning from expository science texts. En M. McCrudden, J. Magliano, \& G. Schraw (Eds.), Text relevance and learning from text (pp. 353-374). Greenwich, CT: Information Age Publishing.

Zwaan, R. A. \& Radvansky, G. A. (1998). Situation models in language comprehension and memory. Psychological Bulletin, 123, 162-185.

Para citar este artículo:

Saux, G., Irrazabal, N. \& Burin, D. I. (2014). Detalles seductores relevantes e irrelevantes en un texto expositivo: efectos sobre la comprensión y el recuerdo en lectores con bajo conocimiento previo.

Ciencias Psicológicas VIII (1): 89 - 100. 